\title{
La sociedad y el especialista, el papel del psicólogo como burócrata de la normalidad: entre la clínica, su desarrollo histórico y sus implicaciones
}

\author{
Jeyson Ariel \\ Ramírez Cortés**
}

jeiar11@hotmail.com
Sebastián Hurtado Jiménez ${ }^{* * *}$

Artículo corto de investigación recibido el 09/01/2018

y aprobado el 10/03/2018

\section{sebastian.hurtado@live.com}

$$
\text { y aprobadoel 10/03/2018 }
$$

तो

Cómo citar este artículo:

Ramírez Cortés, J. y Hurtado Jiménez, S. (2018). La sociedad y el especialista, el papel del psicólogo como burócrata de la normalidad: entre la clínica, su desarrollo histórico y sus implicaciones. Trans-Pasando Fronteras, (11).

\footnotetext{
* El presente artículo se constituye como resultado de un ejercicio de reflexión teórica, llevándose a cabo en el marco del semillero de investigación Lôgos, adscrito a la línea de investigación subjetividad y cultura de la facultad de psicología de la universidad Santiago de Cali. Una versión de este escrito fue presentada como ponencia en el III Encuentro Nacional de Estudiantes de Psicología (ENEPSI), organizado por las universidades Icesi Y javeriana de Cali, Colombia. Cali, 12-13 de octubre de 2017.

** Estudiante de psicología de la universidad Santiago de Cali. Pertenece al semillero Lôgos adscrito a la línea de investigación subjetividad y cultura.

***Estudiante de psicología de la universidad Santiago de Cali. Pertenece al semillero Lôgos adscrito a la línea de investigación subjetividad y cultura.
} 


\section{Resumen}

El presente trabajo apunta a suscitar una reflexión en torno al papel del psicólogo a partir de las problematizaciones que ha implicado la aparición de profesionales que componen las Ilamadas disciplinas en función psi. Para ello, se formula una apreciación a manera de crítica sobre la demanda permanente de las instituciones y las implicaciones que conlleva el papel del psicólogo en estas. Además, se trazarán algunos aspectos históricos que dieron paso a la presencia de profesionales que intervengan en la normalización y promoción de la estructura social, localizando su aparición en función de una sociedad que lo ha demandado. Cabe mencionar que, más allá de lo que podamos proponer como una salida frente a lo que idealmente puede hacer la psicología para evitar la problematización que planteamos, es más bien un llamado a pensar continuamente la psicología, el papel del psicólogo actualmente y promover el ejercicio crítico que para ello se requiere.

Palabras clave: Locura; Normatividad; Orden social; Diagnostico; Sistema. 


\section{Society and the specialist, the psychologist's role as an official of normality: amongst clinical, it's historical development and implications}

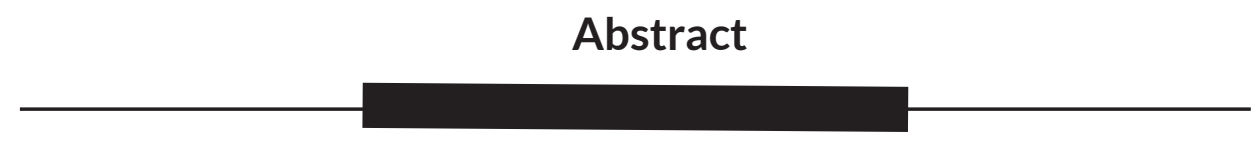

This workaims to incitea reflection concerning the psychologist's role coming from the problematics that have involved the appearance of the professionals that make up the so called disciplines in function of psi. Hence an appraisal is formulated as a critique on the permanent demand of the institutions and the implications of the psychologists in them. In addition, some historical aspects that led to the presence of professional intervention in the normalization and promotion of the social structure inquiring its appearance in function of a demanding society will be outlined. It is necessary to mention that, beyond what we could propose as a way out of what psychology ideally could do to avoid the problematics that we contemplate, it is rather a call to consistently think psychology, the current psychologist's role and to promote the critical practice it requires.

Key words: Madness; Normativity; Social order; Diagnostic; System. 


\section{Introducción}

"No sólo existe solidaridad entre el objeto y los métodos de la psicología sino que un tercer aspecto se suma a esa totalidad integrada: el rol del psicólogo, lo que se espera que él haga".

\section{(Benedito, 1982, p.403)}

Diariamente, personas acuden ante el profesional en psicología aquejándose de una problemática que ha pasado a ser algo perturbador y no le permite tener estabilidad (ya sea física o anímica) $y$, que ante las soluciones planteadas por el mismo paciente no ha sido posible evitar ese displacer que lo trae finalmente a consulta. Sin embargo, lo que se espera del psicólogo en determinados casos es que al final de una consulta (que en una institución de salud podría rondar entre los 15 y 20 minutos) emita una serie de interpretaciones que lo lleven a señalar cuál es la problemática, cómo va a intervenir, en cuántas sesiones y cuáles son los resultados a los que aspira llegar, entre otro tipo de planteamientos. Es decir, que después de escuchar solamente una mínima parte del discurso de una persona, el sistema exige que el especialista -que en este caso es el psicólogo- señale qué es lo que se encuentra mal y qué es lo que este debe hacer. Así, no es de sorprender, que bajo esta misma condición, se le pida un pronóstico, ya que en su objetividad, sabe qué pasará con la singularidad ${ }^{1}$ que atraviesa al ser

${ }^{1}$ Singularidad pensada como término matemático: se usa para aludir a ciertas funciones que presentan comportamientos inesperados. Es decir, lo incalculable. Un punto en el que se asignan valores a determinadas variables de la función, provocando la imposibilidad de cálculo del producto. De ahí que se hable de funciones que tienden a lo infinito. 
hablante. En relación con lo anterior, podemos pensar entonces que plantear la siguiente pregunta al profesional resultaría ser problemática: Cómo el hecho de no llegar a este tipo de resultados pondría en tela de juicio las capacidades y el conocimiento del profesional en psicología.

Lo anterior, se encuentra directamente relacionado con la pregunta por la psicología, que en un momento Canguilhem (1956) suscitó2:

¿Qué es la psicología? parece más embarazosa para un psicólogo que la pregunta ¿Qué es la filosofía? para un filósofo. Pues, mucho más que definirse por una respuesta a esta cuestión, la filosofía se constituye a través del interrogante sobre su sentido y su esencia. Para quien quiera decirse filósofo, el hecho de que la pregunta, a falta de respuesta satisfactoria, renazca sin cesar es un motivo de humildad, y no una causa de humillación. Pero, en el caso de la psicología, la cuestión de su esencia o, más modestamente, de su concepto pone también en entredicho la existencia misma del psicólogo, pues a este, incapaz de poder responder con exactitud qué es, le resulta muy difícil contestar qué hace. No puede, entonces, más que buscar en una eficacia siempre discutible la justificación de su importancia de especialista, una importancia que, si generara en el filósofo un complejo de inferioridad, no disgustaría en absoluto" (Canguilhem, 1956, p.389)

${ }^{2}$ Título original: "Qu'est-ce que la psychologie?, Conferencia pronunciada en el Collége Philosophique el 18 de diciembre de 1956. Se publicó por primera vez en la Reue de Métaphysique et de Morale ,1, 1958, y sereeditó en Cahiers pour l'Analyse, 2, marzo de 1966. 
Por lo tanto pareciera que la dificultad para responder a la pregunta en cuestión, radica en que no existiría del todo una psicología, sino más bien, unas psicologías que en función de encontrar una explicación al acaecer anímico, terminan creando una ideología disfrazada de ciencia. Se enjuicia por medio del aspecto ideológico debido a la fragmentación de la psicología y por el hecho de que el estatuto ideológico se da pretérito a la constitución de una ciencia. En ese sentido, Braunstein (1982) sostiene: "Entre el saber ideológico y el conocimiento científico hay un claro corte (ruptura epistemológica) pero también hay una relación indisoluble que los liga y los implica recíprocamente" (p.11). Así, lo anterior posibilita pensar si realmente la psicología, mediante su fragmentación, se ubica en el marco de lo científico o de lo ideológico, siendo por medio de esa reciprocidad que se pretende sostener la idea de ciencia. Además de alimentar la pretensión de saber, que el título de psicólogo otorga a su vez una tendencia a evitar el no-saber, como si se tratase de algo que no puede ser permitido, como si las respuesta y explicaciones constituyeran algo implícito en la psicología. Es así que, a falta de unidad en la psicología, siendo a raíz del surgimiento de nuevas formas de estudiar y entender lo anímico, surgen nuevas psicologías, contrarias y disruptivas las unas de las otras, tratando de justificar epistemológica y científicamente su existencia. Entonces sí cada psicología responde diferente a lo que es o debería ser, como señala Canguilhem (1956), difícilmente podrá responder a las cuestiones sobre su quehacer.

Más allá de lo que constituiría como tal el ejercicio del psicólogo, el sistema y la sociedad han demandado su presencia, al igual que la de otros profesionales que se encargan de regular y mantener el orden social. Con relación a lo anterior, se hace referencia a un orden donde cada quien debe ser productivo y velar por la seguridad de sus miembros, sobre lo cual se enmarca el papel de 
los diferentes profesionales, procurando que ello se cumpla bajo determinados parámetros. De esta forma, se recrea una trama sobre la cual se exponen ejercicios de poder ${ }^{3}$, desarrollos históricos y problematizaciones que invitan a pensar la psicología continuamente, exhortando la posición crítica de los profesionales y estudiantes de psicología en la actualidad.

\section{El sistema y su demanda continua al psicólogo, implicaciones en el campo clínico.}

En el campo de la clínica, el rol del psicólogo ha sido en algunos casos una exigencia, pero ¿qué exigencia? Podríamos pensar esta como la necesidad de adaptarse a un papel asignado: El que tiene la verdad, el que cura, el que emite una respuesta y una solución viable para la problemática del paciente. La demanda del sistema, de las instituciones y la sociedad lleva a que cada vez sea más frecuente la idea del profesional que se limita a emitir respuestas y juicios equivalentes en defensa de una supuesta eficacia. La eficacia entonces, según como lo plantean las terapias actuales, iría en dirección a la inmediatez, donde no hay cabida para el interrogante, sino para la resolución de sus problemas sin que su esfuerzo se vea involucrado ni sepa lo que se encuentra operando en razón de su proceder. Con ello no se pretende plantear una apología a las terapias de larga duración, sino señalar las cosas que implica el quehacer clínico, más allá de las técnicas de intervención como pruebas psicotécnicas o encuadres con sesiones contadas.

\footnotetext{
${ }^{3}$ Se hace referencia a las diferentes formas en las que se ejerce el poder. Por medio de pruebas, estadísticas, formas de institucionalización, etc. Todas aquellas relaciones donde se enmarca un saber por encima de otro.
} 
Es a raíz de ello que retomamos lo que Pasternac (1982) sostiene sobre el método clínico:

La palabra "clínico/a" nos orienta en nuestra búsqueda. Todos los autores señalan su procedencia del arsenal nocional de la medicina. En ésta lo clínico se refiere originalmente al estudio detallado (hecho por el médico) de un paciente que yace en su lecho (clinos = lecho): forma de aludir al enfermo concreto por oposición a la "enfermedad" como patología abstracta... Efectivamente, lo que caracteriza al método clínico, en una primera aproximación, es el estudio en profundidad y en extensión de un caso. El "caso clínico" explorado en todas sus variables evoca la situación inversa de la del método experimental donde se intenta explorar las modificaciones de una sola variable en multitud de casos que constituyen ya sea la totalidad de un universo o una muestra representativa del mismo. (Pasternac, 1982, pp. 146-147).

Es así que, el hecho clínico consistirá, en un primer acercamiento, en el abordaje de todas las variables posibles de un caso y la singularidad que este implica. No se trata de controlar las variables ni de generalizarlas, como es el caso del método experimental, sino de tener en cuenta que en el momento en que se estandariza un mismo proceder para todos los casos, se pierde el abordaje clínico. Por otra parte, el desarrollo de método clínico ha ido abandonando la idea de enfermedad, sin embargo, lo que mantuvo y por lo que vela es por la singularidad que atraviesa el caso clínico.

Si a partir de aquí pensamos el ejercicio del psicólogo tal y como lo hemos venido planteando, pareciera ser que implica que este es una máquina de resultados, de respuestas, de curas y soluciones, dejando a un lado las cuestiones que tienen que ver con lo propio 
de cada caso. Es así que un retorno a la clínica se vuelve imperante en la psicología. Más allá de las demandas del sistema, retornar a la clínica debe pensar los principios de observación y escucha que implica la singularidad de cada caso.

En el diccionario de la RAE, encontramos una definición muy particular en una de las acepciones para la palabra "papel" (teniendo en cuenta el sentido de la palabra con relación al concepto de rol) que lo define como "Parte de la obra dramática que ha de recitar cada actor y la cual se le entrega para que la estudie". Desde esta definición podríamos pensar entonces que el psicólogo cumple un papel, lo estudia, lo recita y lo actúa, no significa que este sea su proceder ante cada caso particular, más bien se adapta a repetirlo en cada escena, a lo que esperan de él, a una norma, a la regla, al modelo. Curiosamente, al reflexionar sobre lo anterior nos encontramos con una característica repetitiva frente a la concepción de la salud mental y la norma: Ios pacientes reciben la etiqueta de "enfermo" al ser marginados de la norma por la particularidad en su conducta y su forma de relacionarse con su entorno, simultáneamente, el mismo profesional que ha emitido esa respuesta junto a la etiqueta de dicho paciente será cuestionado ante una norma si no asume el rol que le corresponde (si no demuestra con resultados inmediatos lo que esperan que sustente su proceder).

Por esto no es nada raro encontrarnos actualmente con psicólogos que ante esta demanda permanente, no muestran interés en profundizar en las particularidades del caso, en analizar, en ser críticos ante lo que podrían hacer mediante la terapia, sino que pareciera que ya tienen un molde previamente construido, un plan, un método (tal como el médico que se limita a ver la pantalla de su ordenador mientras pregunta los síntomas del paciente 
y termina la consulta sin haber interactuado realmente con la situación), como si el motivo de consulta fuera finalmente lo que se buscara resolver, generando un buen posicionamiento social del profesional (que cree que dio la solución correcta) pero dejando entrever la desconfianza que puede generar su rol actualmente.

En instituciones como la familia o la escuela, sus miembros con poder pueden ejercer violencia no aparente, es decir, violencia simbólica. De este tipo es la que ejerce el psicólogo hacia su "objeto", que es lo que en particular nos interesa, y puede ejercerla porque el estatus que ocupa implica una concesión de poder por parte de la clase dominante: poder técnico. (Benedito, 1982, p. 414)

A partir de esta relación de poder profesional-paciente se establecen una serie de creencias, entendiendo estas como lo plantea Nocera (2009) a partir de una lectura de Durkheim:

Los usos de la noción de creencia guardan en las primeras formulaciones durkhemianas un parentesco claro con dos conceptos recurrentes en su producción: la conciencia colectiva y el hecho social. Esta aparición asociada, advierte al lector que la creencia es parte constitutiva de los fenómenos en sociedad teniendo una relevancia manifiesta para pensar la dimensión ideal o intelectiva que caracteriza a estos procesos. (p. 1) 
Así, se le asigna entonces al psicólogo una posición de poseedor de un saber y de una técnica, por tanto, el rol que desempeña trae consigo una serie de mensajes implícitos (poseedor de la verdad, solucionador de problemas, generador de diagnósticos, entre otros), permitiendo preguntarnos sobre la forma en que el psicólogo hace uso de ese poder cedido por la sociedad, ¿Cómo el juicio del profesional entra a ser determinante frente a "su objeto de estudio" en su momento?, refiriéndonos precisamente a un tema en particular: El diagnóstico, En qué medida esa búsqueda de la cura termina siendo más una condena?

Consideramos que cuando el diagnóstico se basa en una forma de "recitar mi papel como psicólogo" (nos referimos con esto a la idea de emitir un juicio con la pretensión de demostrar que yo como profesional sé qué le sucede a mi paciente, genero una respuesta o veredicto que responda a la demanda, porque no saberlo pondría en tela de juicio mi conocimiento y mi reputación), se consigue, como efecto adverso, posicionar al sujeto en el discurso de la categoría nosológica indicada. Es así que el diagnostico en tales casos pasa a ser una forma de identificación con la cual el sujeto trata de orientarse. Es decir que, al sacar al sujeto del discurso social e introducirlo en el discurso del trastorno, se promueve el despojar la palabra del sujeto o desterrarla en función del orden social.

No obstante, lo que nos compete no es problematizar todo lo que es asunto diagnóstico, sino las implicaciones que tiene este en el momento en que el profesional hace uso del poder cedido por el sistema para emitir una respuesta que se demanda, teniendo en cuenta que una de las exigencias permanentes en el sistema social de salud, es la emisión de un juicio a partir del cual haya evidencia de que se está realizando algo por la persona. Interesa mostrar 
resultados y llevar todo un papeleo que certifique la permanencia de la institución y sus profesionales, más allá del abordaje a cabalidad que conlleva un ejercicio complejo e interdisciplinario.

\section{La lógica de intervención frente a la anormalidad desde sus as- pectos históricos en torno a las exigencias sociales.}

Teniendo en cuenta entonces lo que desarrollamos como rol o "papel" del psicólogo, planteamos de antemano trazar una analogía con la concepción de la psicología social que Benedito (1982) retoma de lo que es una sociedad concibiéndola como una "compleja organización de posiciones". Sobre lo cual, podríamos pensar que el rol-papel del psicólogo se debe, en gran parte, a una posición social otorgada, a un papel que lo posiciona y el cual reproduce para mantenerse en un status, y a su vez, otorga al que está delante de su escritorio una posición, en la que busca orientarse dentro de la sociedad. (pp. 103-115)

Asumimos entonces, que lo que respecta al rol y su posicionamiento social, son aspectos inseparables para tener en cuenta en el quehacer del psicólogo en la actualidad, de ahí no solo se desprenden las cuestiones entorno al sistema que hemos explayado, sino que, para la contemplación de esta trama, se deben pensar las cuestiones sociales que dieron lugar a todo ello. Es aquí, donde tras haber planteado las vicisitudes referentes al rol del psicólogo y las demandas que le impone el sistema, nos remitimos a las demandas y fenómenos sociales que terminaron precediendo a lo que actualmente consideramos como profesional en el campo de las Ilamadas disciplinas en función psi. En relación con los aspectos histórico-sociales que queremos introducir, Canguilhem (1971) sostiene que: 
Existen condiciones objetivas que nos remiten a una determinada formación social, que sobredetermina una demanda, un encargo social, que se caracteriza porque debe permanecer implícito: que no tengan que actuar los aparatos represivos del Estado para que cada sujeto del proceso productivo ocupe el lugar asignado en la estructura. (p. 120)

Hablamos entonces de una condición histórico-social que ha permanecido implícita, donde el sistema estatal no se hace evidente sino que son los mismos individuos que regulan su organización social. A partir de esta organización social inician los posicionamientos asignados en función de la permanencia de su estructura, donde el psicólogo se ubica. Es bajo esta condición entonces que la sociedad acude al profesional de la salud, del que espera una respuesta dotada de verdad y ser orientado por la misma, más allá del origen o las características de dicha demanda, se preocupa porque no se encuentre alterado el orden de esta. Es así que planteamos contemplar la cuestión del quehacer interventivo mediante una sociedad que atraviesa una historia que ha demandado profesionales, que operen en función de su organización colectiva. En ello se enmarca toda una lógica que deja en evidencia la exigencia que solicita la sociedad para exiliar a los individuos que alteren su orden.

La sociedad se remite con toda buena conciencia al médico para que éste señale cuáles son los sujetos que deben excluirse por medio de un diagnóstico cuando no es posible integrarlos a cualquier precio a la "normalidad", pero no se interroga antes sobre las significaciones que tienen esas locuras o esos retardos. (Mannoni, 1981, p.12) 
La autora nos advierte que el problema no es específicamente político, lo que se revela en cuestión, no son tanto las formas administrativas que se generan por parte de las grandes elites, sino que es la posición de la mentalidad colectiva ante la anormalidad. De esta forma, la puesta en escena de este panorama proyecta que la cuestión es de orden social. Basta con observar la intención del inicio de los manuales de diagnósticos mentales. El manual clasificatorio internacional de enfermedades (CIE) tiene su origen en la Nomenclatura de enfermedades de Bertillon (1893), sin embargo, es a partir de su sexta edición (1948), que los consultores de la CIE incluyen un capítulo, el quinto, dedicado a las enfermedades mentales (Requena y Jarne, 2015).

Por otro lado, el manual diagnóstico y estadístico de trastornos mentales (DSM), publica su primera edición en 1952. En consideración, Tanto el capítulo quinto dedicado a las enfermedades mentales de la CIE, como el DSM constreñido precisamente a estas, surgen en contextos de postguerra, a partir del cual los profesionales de la salud empezaban a tratar con casos de difícil cabida en las clasificaciones de la época, especialmente trastornos agudos y psicosomáticos (Requena y Jarne, 2015). Toda una expresión de la sintomatología de la época y la permutación del síntoma en sus fauces. A causa de ello se evidencian las repercusiones a nivel social y científico que produjo la guerra en este ámbito de los padecimientos mentales, con lo cual se hizo necesario reajustar el modelo médico para brindar algún tipo de solución a la problemática. La sociedad y las comunidades científicas, exigen que haya una regulación de los individuos afectados por la guerra por medio de un diagnóstico, procesamiento e intervención, y en caso de ser necesario... exclusión. 
Ahora bien, a partir de lo anterior se encierran una serie de problemáticas alrededor de la posición social frente a la sintomatología de época: qué ocurrirá con la salud mental colombiana en el venidero periodo de post-conflicto. El planteamiento anterior no solo pone en tela de juicio la cuestión por una demanda social al especialista en salud mental, sino también la cabida del razonamiento por la propuesta actual de los retos de la psicología colombiana en la actualidad. Así, surge nuevamente el llamado a la reflexión y la criticidad por parte del psicólogo en formación, ya que este periodo de transición y conciliación por parte del gobierno colombiano y los movimientos armados al margen de la ley, tendrán repercusión en los múltiples ámbitos de conformación social y mental. Ello conlleva a pensar una sintomatología de época y los tratamientos posibles de las personas que han estado envueltas en el conflicto armado y estarán compartiendo espacio en nuestra cotidianidad. Los retos del psicólogo colombiano circulan alrededor de todas las cuestiones que se desprendan de ahí.

Por otro lado, la demanda permanente de la sociedad y su concepción de la normalidad, tienen toda una historia que las sustenta. Si se analiza detenidamente, se encuentra que cada sociedad ha ido construyendo sus espacios de exclusión; espacios acuñados por sus ciudadanos con la finalidad de mantener a los individuos que se encuentran por fuera de la norma, al margen. Así como Mannoni (1981) encuentra que existe una tendencia por parte de la sociedad a aislar a los individuos a-normales, Foucault (1966) soñaba y a la vez añoraba una ciencia que estudiara estos lugares creados por la "civilización", lugares cargados de significado, que se oponen a otros, que están destinados a borrarlos, a neutralizarlos o a purificarlos, designándolos así, como heterotopías: "Los lugares que la sociedad acondiciona en sus márgenes, en sus playas vacías que la rodean, son más bien reservados a los 
individuos cuyo comportamiento es marginal respecto de la media o de la norma exigida" (Foucault, 1966, p. 23). Esto abre un panorama a revisar en la historia de la civilización y la exigencia que ha tenido la sociedad frente a los individuos que alteran su orden. Para ello nos servimos del concepto Heterotopía, formulado por Michel Foucault (1993) para dar cuenta de que aquello que conocemos como: instituciones mentales, prisiones, orfanatos, casas de reposo, son lugares privilegiados por ser producto de la "organización social" y bien común. Es, a medida que van surgiendo estos otros lugares acondicionados por la sociedad, que surgen especialistas que hacen cumplir las pautas por las cuales los individuos son procesados y ubicados, o más bien, reubicados.

A manera de ejemplo, cabe retomar el desarrollo de la historia de la locura de Foucault (1993) en torno a los Ilamados "insensatos". En el siglo XVII, eran caracterizados por evidenciar un desarreglo de sus costumbres, evidenciando una modificación en torno a la norma, que ubicaba a estas personas como "desordenadas" al encontrarse por fuera del orden moral. Explicación atribuida a una particular "debilidad del alma" y una alienación del espíritu, que desencadenaría en la vida cotidiana del sujeto por medio de una fenomenología marcada por el escándalo, sacándolo de lo normativo en cuestión. A raíz de ello, es imperante conocer la forma de proceder frente al "insensato" antes del siglo XVII y después del XVIII. Hasta el siglo XVII, es decir, abarcando la mayor parte de la edad media, el acto de insensatez y la fechoría por medio de este era dispuesta a confesión.

Cuando alguno de los habitantes incurría en comportamientos que escapaban a la cotidianidad y generaba escándalo, era expuesto al público obligado a confesar su delito, teniéndose la certeza de que el mal, con todo lo que puede tener de violento e inhumano, no puede compensarse ni castigarse si no es expuesto a la luz del día. De esta 
forma, tanto él como su familia quedaban marcados, siendo su delito haber alterado el orden social.

Ahora bien, los procedimientos de enjuiciamiento de la alteración del orden social por medio del acto del insensato cambiaron a partir del siglo XVII. A partir de este siglo lo que se llevó a cabo fue el confinamiento de las personas que incurrían en perturbar la normalidad por medio de lo que Foucault (2000) describió como "escandalo", expresión que se atribuía al actuar del sujeto en cuestión. El planteamiento de la época era que tanto el escandalo como el mal que de él emanaba era contagioso y susceptible de ser transmisible en dichos momentos de expresión, por lo tanto, la forma de proceder ahora sería el confinamiento en función de que el escándalo no se siguiera manifestando ni alterará el bien social.

Teniendo en cuenta lo anterior, se puede ver que lo mismo ocurría con los leprosos en la sociedad del siglo XVII y que Foucault (2000) señalaba como "La expulsión de esos individuos hacia un mundo exterior, confuso, más allá de las murallas de la ciudad, más allá de los límites de la comunidad" (p.50). Con lo cual se retoma nuevamente la función de la heterotopía en cuestión como lugar acondicionado al margen de la sociedad procurando la ausencia de los individuos presuntamente anormales, ya que tanto la contaminación de lepra como de insensatez, fue mejor mantenerla al margen de la comunidad.

Así mismo, lo reconocía Canguilhem (1971) "Estar enfermo significa ser perjudicial o indeseable o socialmente desvalorizado, etc."(p.88). De ahí que la sociedad tenga casas de reposo, clínicas psiquiátricas y prisiones, o como se deseen Ilamar, la realidad de la condición del sujeto sigue siendo la misma. Y es esta misma sociedad quien ha pasado de ubicar a un sacerdote a ubicar un "especia- 
lista" que opere haciendo cumplir las pautas de buena conducta, como impartidor de la normalidad. Es decir que este ser perjudicial e indeseable, se encuentra a merced del especialista, quien en función de la sociedad hace valer ciertos criterios de adaptación, que en caso de someterse a estos, podrá "entrar de nuevo en las filas de los bien pensantes".

\section{Conclusión}

Como hemos visto, el rol del psicólogo pareciera que fuera de agente público en función de una sociedad que demanda la presencia y ausencia de ciertos individuos que atenten contra la estabilidad de su organización. Para darse cuenta de ello, solo hace falta contemplar la lógica del estado occidental, por ejemplo: En la escuela primaria nos enseñaron que portarse bien y hacer caso nos evitaría problemas, en la secundaria que había que estudiar para ser alguien en la vida, y en la adultez que hay que ser productivo para ser exitoso. Evitar problemas, ser alguien en la vida y ser productivo, son indicadores de que para poder estar en el contexto sociocultural, implícitamente se debe procurar la productividad estatal y el bien social, de lo contrario la sociedad te asegura otros espacios (la prisión, el manicomio y el cementerio). Con ello entrevemos la cultura, lo que vemos aquí y allá, producto del lenguaje, es decir, lo que llamamos realidad, siendo denominado por sociólogos y antropólogos como ficción, una ficción donde el psicólogo se ha abierto paso entre campos de saber. Lo anterior teniendo en cuenta la definición de cultura que Sampson (2000) propone: 
La tesis que quisiera proponer es que el tercer mundo popperiano, constituido por un conjunto heteróclito de sistemas semióticos, puede válidamente considerarse como ese dominio de realidad objetiva que los antropólogos, los psicólogos culturales y los etnopsicoanalistas denominan "la cultura". Es esta dimensión la única que puede mediar entre el primer mundo de los objetos físicos y el segundo mundo de la representación mental.

Es así que en esta dimensión, entre los objetos físicos y las representaciones mentales, se enmarca, el molde sobre el cual el psicólogo opera como agente cultural. No obstante, este es un llamado a conmocionar y cuestionar el mundo de la psicología e ir más allá de lo que la sociedad demanda como resultados objetivos y estudiar la particularidad de cada caso, reflexionar sobre cada sujeto y pensar su quehacer en torno a una autocrítica por medio de la epistemología y la complejidad que demanda el caso por caso.

En ese sentido, es menester retornar a los fundamentos de la clínica con mayor importancia en la actualidad, cuyo periodo por venir alrededor del post-conflicto demanda como reto a la psicología. Así, la observación y la escucha como herramientas indispensables para el profesional en salud en el momento de ejercer su rol/papel, junto con lo que implica la mirada clínica, es lo que permite mantener una postura crítica acerca de cada caso y las posibles evidencias dentro de los mismos, ya que consideramos que es algo que paulatinamente ha ido desvaneciéndose, y en su lugar han llegado otro tipo de métodos (véase estadísticas, estándares, signos y síntomas no ligados a un discurso ni a una imagen, test psicológicos, etc.) que en su momento habrían sido propuestos 
como una ayuda o material de evidencia en determinados casos, pero que en algunas situaciones de la actualidad han pasado a ser un producto generador de etiquetas para quien lo realiza, incluso reemplazando el criterio del profesional (donde los resultados de una prueba no dejan lugar a ninguna objeción), pasamos a estar dentro de un sistema que observa un sujeto completamente estadístico (Cl bajo/alto, nivel de depresión, nivel de demencia, etc.), que no ha sido escuchado más que en una sesión, pero si rotulado tras una evaluación escrita, y que tampoco ha sido observado en profundidad porque lo que realmente importa reside en el resultado en las pruebas psicotécnicas. Sin embargo, es para nosotros de gran importancia abrir la discusión acerca de los aspectos que podrían conducir a repensar lo que podría hacer el psicólogo ante las demandas del sistema y la sociedad, considerando que dicha labor debe superar las demandas impuestas. Es decir, más allá de las respuestas que tanto el sistema como la sociedad demanden, lo que debe imperar es la exploración de cada caso de forma particular, teniendo en cuenta el discurso que pregona cada sujeto lo enmarca en el campo de la singularidad. Entre las interrogantes que se abren a partir de esta propuesta podría estar la de si el psicólogo debe, entre su quehacer, renunciar a creer que sabe, y así afrontar cada caso de una forma distinta, lo cual le evitaría pretender solucionar de entrada cada problemática que se le presenta como una demanda de saber, llevándonos a recordar a Bercherie (1980) quien afirmó:

Por una parte, lo que se fijó en forma de hipótesis, teorías, principios, como aquello que parece necesario para hacer comprensible la experiencia, tiende a desarrollar sus consecuencias lógicas y a suministrar las ciencias deductivas que se anticipan a la experiencia. Por otra parte la experiencia así posibilitada termina por desbordar 
los marcos, dando resultados cada vez menos conciliables con sus premisas teóricas, y los cambios de hipótesis o de teorías que se imponen pueden llegar a estremecer lo que parecía un principio definitivo, necesario o a priori de la razón. (p. 10)

Es así, que lo que se plantea no es prescindir totalmente de todo el sustrato teórico de los profesionales, sino tener en cuenta que la realidad se escapa al manual de intervención, ya que esta simplemente se construye como un modelo para tratar de dar explicación a un fenómeno o una serie de ellos. No obstante, el caso humano, como ya lo hemos comentado, se escapa a toda posibilidad de control sobre sus hechos, ya que en él habita una condición singular que lo separa de la posibilidad de estandarización. Finalmente nos encontramos con propuestas alrededor de la forma de concebir la salud mental en la actualidad, basándonos en la ley colombiana que pareciera no incluir los aspectos históricos y subjetivos de cada sujeto, y su influencia sobre la salud mental, entre varios aspectos que planteamos formular en una reflexión hacia lo que hoy consideramos es el quehacer del psicólogo. Por lo tanto cabe retomar dicha ley:

La salud mental se define como un estado dinámico que se expresa en la

vida cotidiana a través del comportamiento y la interacción de manera tal que permite a los sujetos individuales y colectivos desplegar sus recursos emocionales, cognitivos y mentales para transitar por la vida cotidiana, para trabajar, para establecer relaciones significativas y para La Salud Mental es de interés y prioridad nacional para la República de Colombia, es un derecho fundamental, es tema prioritario de salud pública, es un bien de interés público y es componente del bienestar general y el mejoramiento de la calidad de vida de colombianos y colombianas. (Ley No 1616, 2013) 
El hecho de no incluir los aspectos históricos y subjetivos del sujeto frente a la concepción de la salud mental le arrebata una parte crucial a su situación, es decir, que se concibe una salud mental parcialmente incompleta, ya que se omiten dos puntos claves para la construcción de la misma, se hablaría entonces de un sujeto que se manifiesta en la cotidianidad pero sin un pasado, sin una elaboración previa de identidad subjetiva.Debemos tener en cuenta entonces que la historia constituye, una gran parte de la puesta en acto del sujeto, además de que almacena contenido que permanece en el aparato psíquico, es decir, que muchas de las señales acerca de su salud mental se deben a condiciones que la historia le ha otorgado, y por ende el hecho de omitir este aspecto nos dejaría simplemente con una serie de comportamientos e ideas no ligadas a un pasado ni a un contexto histórico determinado, aspectos que además se han encargado de construir parte de su identidad y que de forma inminente son causales de lo que actualmente podemos evidenciar.

La subjetividad, por su parte, refiere al caso por caso, lo que podría remitirnos a que el concepto de salud mental para la ley colombiana no abarca contenidos referentes a lo que podría ser un malestar subjetivo, en relación también el padecer de cada sujeto, lo cual evita toda posibilidad de establecer un parámetro general o un manual universal de salud mental. Por lo tanto, si a ello sumamos que la historia a nivel mundial es cambiante entre cada población, es mucho más complejo el pensar una definición global de salud mental a partir de lo subjetivo, ya que estos aspectos que son de trascendencia para la construcción de esta, no están siendo apreciados, y de ser así, de forma irónica, imposibilitarían el pensarse una definición que logre abarcar totalmente una idea de salud mental que represente la realidad de cada población. De igual forma vemos cómo la ley colombiana de salud mental prefie- 
re ahorrarse el trabajo de examinar la historia del sujeto, ya que lo que se entreteje en sus intereses tiene que ver más con la funcionalidad del sujeto en el "aquí y el ahora". Es así, que pareciera que la salud mental estuviera encaminada, al igual que Colombia, a borrar su historia. En función de lo anterior, es que se propone mantener una postura crítica no solo frente al psicólogo, sino frente a la salud con la cual se constituye gran parte de su quehacer, Invitándolo a pensar nuevamente el sistema de salud mental, tal y como se elucubra en el interés colombiano.

\section{Bibliografía}

Benedito, G. (1982). Rol del psicólogo: rol asignado, tol asumido y rol posible. En Braunstein, N. (Ed), Psicología, ideología y ciencia. (pp. 103-115). Madrid: editorial siglo XXI.

Bercherie, P. (1980). Los fundamentos de la clínica. Paris: Navarin editeur.

Braunstein, N. (1982). ¿Cómo se constituye una ciencia? En Braunstein, N. (Ed), Psicología, ideología y ciencia. (pp. 7-21). Madrid: editorial siglo XXI.

Canguilhem, G. (1956). ¿Qué es la psicología? Buenos aires: Amorrortu editores.

Canguilhem, G. (1971). Lo normal y lo patológico. Argentina: editorial siglo XXI

Foucault, M. (1993). Historia de la locura en la época clásica [Vol. I]. Ciudad de México: Fondo de Cultura Económica.

Foucault, M.(2000).Losanormales. Argentina:fondodeculturaeconómica 
Jarne, A. y Requena, E. (2015). Sistemas de clasificación y diagnostico en psicopatología. En Jarne, A. (Ed), Manual de psicopatología clínica. (pp. 75-108). Barcelona: Herder

Ley N 1616, Diario Oficial del congreso de la república, Colombia, 21 de enero de 2013.

Mannoni, M. (1981). El psiquiatra, su loco y el psicoanálisis. Madrid: editorial siglo XXI

Nocera, J. (2009). El concepto de creencia en la sociología durkheimiana. XXVII Congreso de la Asociación Latinoamericana de Sociología. VIII Jornadas de Sociología de la Universidad de Buenos Aires. Asociación Latinoamericana de Sociología, Buenos Aires.

Pasternac, M. (1982). El método experimental y el método clínico en psicología. En Braunstein, N. (Ed), Psicología, ideología y ciencia. (pp. 127-146)

Sampson, A. (2000). Mente, cultura y enfermedad. Revista Colombiana de Psicología, O(9), 23-31. Recuperado de https://revistas.unal.edu.co/index.php/psicologia/article/view/16108/16994 